\title{
Persistence and Lurkers in Discussion Lists: A Pilot Study
}

\author{
Blair Nonnecke \& Jennifer Preece \\ Information Systems Department, University of Maryland Baltimore County \\ 1000 Hilltop Circle \\ Baltimore, MD 21250 \\ \{nonnecke,preece\}@umbc.edu \\ Nonnecke: (410) 233-5771 \\ Preece: (410) 455-6238
}

\begin{abstract}
In email-based discussion lists (DLs), messages resident in archives, email clients and elsewhere are persistent. One way of examining persistent messages is through the eyes of lurkers. For participants in this study, persistent conversation is an inhibitor to participation, a mechanism for engendering participation, and something to be managed. Participants viewed persistent conversation, especially when it can be retrieved through search mechanisms at a later date, as a loss of security and privacy, and an impediment to public participation. Participants followed conversations to understand the practices and language of a DL. Strategies for reading and managing email were idiosyncratic and goal driven. Some participants were concerned about maintaining access to DL information for future use. Others, more concerned about being overloaded with too much email, focused on eliminating messages. Where possible, design implications are put forward.
\end{abstract}

\section{Introduction}

Although lurkers are in the majority in DLs [4, 8], little research has been forthcoming on what they do and why. Apart from several academic works $[5,6]$ and an online column [4], lurkers in online discussion groups have received little attention. To better understand lurkers and lurking, we are developing a model of lurking that includes the following elements:

- personal forces within the individual lurker

- characteristics of the medium of email

- characteristics of email based discussion groups

- characteristics of dialogue within a DL

- tools and their influence

- activities of lurking.

Persistence as it affects lurkers falls under most of these elements, but most obviously as a characteristic of the medium of email. This paper concerns itself with understanding how persistence affects lurking and in particular how lurkers view it as a benefit to lurking, as a hindrance to public participation, and as overhead.

To put the discussion of lurkers in context, the following definitions may be helpful. The Jargon Dictionary [3] defines lurker as:

One of the 'silent majority' in a electronic forum; one who posts occasionally or not at all but is known to read the group's postings regularly. This term is not pejorative and indeed is casually used reflexively: "Oh, I'm just lurking." When a lurker speaks up for the first time, this is called 'delurking'.

In contrast, Merriam-Webster's WWWebster Dictionary [9] provides a pejorative definition for lurk:

$\mathbf{a}:$ to lie in wait in a place of concealment especially for an evil purpose $\mathbf{b}$ : to move furtively or inconspicuously $\mathbf{c}:$ to persist in staying

These definitions provide divergent perspectives and reflect an inadequate understanding of the lurker. The Jargon Dictionary definition evokes the image of a benevolent yet responsible Net citizen, while the traditional definition implies something much more sinister.

For the purpose of this article, a lurker in a DL is defined as anyone who for prolonged periods receives communications without publicly posting. For many this may mean never posting in some DLs. We view lurking without a pejorative or negative connotation, but as a means of participating in a DL, albeit without public posting. The goal of this research is to improve our collective understanding of lurkers, their activities, and the tools they use. Persistence is a key element in framing our understanding of the lurker. 


\subsection{Persistence of email in DLs}

L-Soft, one of several suppliers of email list management software, indicates there are over 55 million list members and over 150 thousand public and private lists using their software [7]. Their figures imply that DLs are widely used and cover a broad range of topics.

DLs facilitate delivery of email to a set of subscribed members using a broadcast model. Anyone who sends email to the central server effectively broadcasts the email to all members of the DL. Individuals can respond to received email via the server, which in turn broadcasts the reply to all members. There may be an intermediate step in which messages are moderated. This can introduce delays in propagation and/or the elimination of some email depending on how the moderation is handled. At the member's option, the email may be received individually or in digest form (containing a group of messages). For the most part, DLs are automatic devices for sending and receiving messages amongst members. DLs are asynchronous communication tools in which members can choose when to view their messages, if at all.

As persistent correspondence, email from DLs may be found in many places, some private, some public, and some corporate. These include the receivers' computers, of which there is typically one per member, and often times in a central archive. Email may also be intercepted or backed up and held in corporate databases. Being both persistent and dispersed means DL messages are searchable and manipulable by many.

Whether as single emails, digests, or archives, DL messages contain header information that includes sender, date, and subject. The header information also allows messages to be sorted and managed through various software, e.g., following a thread by sorting messages by the author, subject, and date headers in an email client such as Eudora. In addition, each message contains content and frequently a signature. Given the ubiquitous residences, the messages make great fodder for searching. Searching can range from a member searching her/his own locally maintained email to a researcher searching for quoted text through the use of crawler-based search engines, e.g., Excite [1].

Because copies may reside in many locations outside of the subscriber's control, access is effectively wide open. The messages can be searched for content, originator, or in many other ways. DL email may be read in isolation and the flow and intent of the messages can be distorted through the redistribution of individual messages or parts of copied message. For all intents and purposes, email from DLs may be mutated from dialogue to data and back to content, without the originator having control over the process or use. Examples include the trolling of DLs for the creation of address lists, which are then sold to spammers and legitimate businesses. The copies may also be used in the way they were intended, e.g., as an accessible resource for the group, for finding specific information, and for following conversations.

A number of factors affect how individuals manage their DL email, e.g., total number of messages, their rate of receipt, age, and size. DLs vary in the quality of messages, the number of members, content, topic, terminology, etc. All affect the ways in which the messages are dealt with by the receiver, and thus the level of persistence the messages have at a local level.

In addition to the primary artifacts (the messages, digests and archives), there are often related Web sites, sets of frequently asked questions (FAQs), and electronic forums such as chat rooms and bulletin board systems (BBSs). The principal focus of this paper is on persistence as it relates to the primary conversational artifacts.

\subsection{Design issues}

The persistent artifacts of DLs, the email messages, digests and archives are viewed and manipulated with a variety of tools, from UNIX mail to integrated Web browser-email-news reader tools. Archives themselves can be downloaded as text or, as is frequently the case, imbedded in a Web-based user interface (UI). As part of a larger study, the authors' current interests lie in email clients and not the Web-based UIs to archives.

Email clients have various levels of functionality and usability and have changed over the years, the most obvious differences being found in the graphical user interfaces (GUIs). There is little evidence of email clients being designed for the purpose of dealing with email from DLs, i.e., they were not designed for the volume of messages or the variety of activities associated with belonging to a DL or multiple DLs.

The differences in email clients, and the fact that these tools are being used for the more general purpose of sending and receiving email, means that no two users will see DL emails or act upon them in an identical fashion. For example, while some users will use filters to direct messages to DL-specific mail boxes, others deal with all messages in their primary inbox. In the latter case, DL messages are intermixed with other types of email. It is at the email client and to a lesser degree the server software, where many of the design implications will be discussed.

There are design issues for other areas such as server administration. How a DL owner administers a DL has an affect on the lurking process. For example, DL owners determine whether DL content is delivered as a digest or as separate emails. Lurkers themselves have a profound affect on the quality of their experience; individuals with high levels of expertise with their email client may be 
better able to receive higher volumes of email or capable of belonging to a greater number of DLs.

\section{Method}

Our goal in this work was to develop a preliminary understanding of lurkers and lurking. As such we were looking for a method that would reveal as much as possible in as rich a way as possible. Our method borrows from Nielsen's discount usability [10] in that we were interested in gaining as much insight as possible given our resources. It also draws from Contextual Design [2] in the way the information was gathered and analyzed. This pilot study forms the basis for a more detailed examination of lurking in DLs. Results from a follow-up demographic study can be found in Nonnecke [11].

Lurkers have received very little attention, and as this was our first study of lurking, we assumed, rightly or wrongly, that lurkers would not respond to either email or Web-based surveys. We also surmised that if lurkers did respond we would get a biased response without easily understanding the nature of the bias. More importantly, we were interested in a richer understanding of lurking than could be derived from surveys. However, email and Web-based surveys have shown potential in other studies [15], and will likely be employed in our follow-up work.

Given the relatively high incidence of lurkers, we felt comfortable selecting participants at random from physical communities in which members were known to be Internet users. Ten interviewees were drawn from two locales, 5 men and 5 women, ranging in age from early 20 s to early 50 s. Our intention with the small sample size was to balance for age and gender, rather than examine age or gender issues. All participants were members of at least one online group, and were not pre-selected for lurking or for their level of experience with online communities. All persons asked participated in the study; 3 were well known to the researchers, and 7 were not.

Face-to-face or phone-based, open-ended interviews lasted between 45 minutes and 2 hours, and focused on the interviewee's participation in online groups. Prompting was minimal, and the interviewer did not validate whether a group or topic was worth discussing.

\section{Results and Discussion}

The participants described 41 communities of which there were 25 DLs, 7 BBSs, 5 newsgroups, 3 chat rooms, and $1 \mathrm{MOO}$. All participants belonged to or had belonged to communities in which they never posted, or posted rarely, e.g., once or twice, or so infrequently that they considered themselves to be lurkers. All participants had posted in at least one of their online communities.
The participants in this study were all aware of issues surrounding persistence of email without necessarily having thought about persistence as a factor affecting their actions. Many of the comments made during the interviews were related to other issues, and not persistence per se, e.g., time available, minimizing effort, privacy, entertainment value, and searchability. Recognizing that many of these issues are related to persistence, we examined our interview data from the perspective of persistence, breaking the analysis into three primary areas as it relates to DLs:

- persistence as an aid to lurking

- persistence as an incentive to lurk

- persistence as overhead to lurking.

These areas were chosen for several reasons. With respect to the first area, we view lurking as a form of participation, and want to understand how persistence affects lurkers in a positive way, i.e., as an aid to lurking. In a previous paper [12] we described lurking as a means of satisfying goals. However, we recognize that lurking is not merely goal satisfaction and that other influences are at play. Our second area represents an attempt to understand how persistence acts as an incentive to lurk. The third area reflects our understanding that lurking is work, and like any other activity has overheads associated with it. If we can identify these overheads, then we may better understand the design implications.

\subsection{Lurking Experience}

To provide a flavor of the participants' lurking, two participants' experiences are presented. The first describes the impact of receiving messages in digest form and the second describes the general lurking process of a participant.

Cathleen's lurking using digests. Cathleen is a well known member of high standing in a professional organization. She is also a very private person. Having a health problem, she sought out and subscribed to an online DL specializing in her health problem. She read and saved all messages in digest form for several months but found it difficult to follow threaded conversations. Some digests were printed to aid reading and to save important messages. After the initial period Cathleen started skimming the digests, reading individual posts based on the subject heading. As her health improved, the DL became less important to her. Throughout her membership, she found the moderation to be aggressive and disruptive. She was eventually removed from the DL by a moderator who falsely accused her of cross-posting.

Fred's general lurking strategy. Fred is a knowledgeable, long-term user of DLs and is a 
technically sophisticated group member. He has belonged to a variety of DLs for reasons ranging from professional to personal interest. As a general rule, he does not post to DLs, preferring to post directly to individuals based on their public posts. He belongs to personal interest DLs to learn about the communities and for entertainment.

Fred follows threads but does not read every message in the thread. If he is very busy, he will delete messages without reading them, confident that the same issue will arise at a later date. When investigating a particular message, he uses the subject header and reads the first paragraph before continuing on. He reads to discover others' problems (e.g., technical problems with software), and says it is difficult to find this type of information in any other way, i.e., it is hard to ask about a specific problem when you don't know the problem exists.

$\mathrm{He}$ is also interested in learning about the community, stating that learning about the members helps him to learn about the community. He systematically described his method of coming to know members:

- information is gleaned from email address, name, signature, and URL

- understanding the members comes from what each says and how it is said

- inferences can be drawn from the choice of a false name

- knowledge about posters' habits comes from their frequency of posting and the time of day they post.

On joining a new DL, Fred reads every message to get a broad sense of the DL. He looks for cross-posts as they tell him how members view the DL in the context of related DLs and newsgroups. DL rules describing topics, moderation policy, and membership requirements, etc. tell him much about the community. Likewise, what members say and how they say it is also informative.

Each of the following three sections contains a series of lurkers' goals drawn from the participants' description of their lurking in DLs. For each goal design implications are described.

\subsection{Persistence as an aid to lurking}

This section describes how persistence was found to help lurkers:

- understand the DL

- satisfy personal needs

- satisfy information needs.

Goal: Understand the DL. Participants described the process of understanding the DL as a period of intense reading of most, if not all, posts. This occurred whether the posts were available as separate emails, digests, or archives. In several cases, individual posts were supplemented by searching and reading archives. During this period, which ranged from days to months, participants worked at identifying the topic or topics of the DL and determining whether this was a good fit for their needs.

Participants also worked at understanding the character of the DL. They did this to increase their understanding of the DL and to become more comfortable with the possibility of submitting messages to the DL, or in several cases side posting to individuals. Character is used very loosely here, and includes:

- terminology or special language

- posters (players and archetypes)

- rules (implicit and explicit)

- responsibilities related to being a member of the DL (implicit and explicit)

- style(s) of interaction, e.g., confrontational, humorous, etc.

- response of members to delurkers

- style and intrusiveness of moderation

- response time to messages

- volume of postings.

Participants' intentions with regard to public posting generally varied from DL to DL. For example, a participant may have joined a DL with the intention of observing and never posting from the outset. If there was a mismatch between their expertise and that expressed in the DL, then this initial period of lurking was used to confirm this before unsubscribing or remaining subscribed but completely uninvolved in the DL. If there was a possibility of posting publicly, they used this period to gauge whether their posts would have value. They also observed whether they would be able to add value, and whether the value, they could contribute, already existed in the DL, i.e., postings by others would make their contribution redundant.

New DL members are inquisitive and DL owners need to take advantage of this. The following are some of the information types that could prove valuable:

- terminology dictionary

- rules, if any

- selected highlights from the archives

- selected personal stories, e.g., in health support DLs

- description of moderation (and moderators)

- topic lists

- message rate

- number of active posters

- number of members.

While some of the above are provided in the subscription or welcome message, messages of this type were frequently unread by the participants. Key to the success of this type of information is making access obvious, timely, and ubiquitous. Many of the above list items could be kept in a DL-related Web site. A link to the Web 
site appended to each DL message could provide access to the site. Unfortunately, having a related Web site and linking back to it is not widely practiced.

Goal: Satisfy personal needs. When DLs were joined for personal reasons there was a correspondingly strong motivation to get as much out of the DL as possible. Entertainment was a common theme and took a variety of forms. Just as some people enjoy receiving snail mail, several participants enjoyed receiving email, indicating they liked having new email in their inbox. This gave them a sense of connection and also something to do in their free time. Others mentioned being attracted to controversy and debate, including watching flaming from the sidelines. Humour was also appreciated. Curiosity and learning were high on many peoples' list of reasons for joining and lurking in a DL.

Others joined DLs with many of the same members as their non-electronic based organizations. In their opinion, this complemented and strengthened relationships. DLs also provided a convenient way to track events and announcements. One participant, who belonged to such a DL, read all messages and deleted all but the announcements for physical meetings.

Some participants are attracted to health-support DLs as a source of empathy [14]. For at least one participant, empathy was strongly felt while lurking. DLs can also act as a mechanism for putting people in contact with one another through more private channels. For example, peers, expertise, and finding people beyond a local geographic community were described as reasons for joining a DL. Topics of specific interest to participants also drew them into joining DLs. Participants often described members of DLs as interested and focused. Relationships developed out of belonging to the DL, although no long-lasting friendships were reported as found elsewhere [13]. Several participants indicated they developed a sense of community through lurking.

For some, persistence in the form of archives provides a sense of security, allowing them to search or review a full set of messages. Having access to such an archive appears to reduce the fear of missing something.

Members of DLs have a variety of personal needs to satisfy. These are far ranging and a number of different approaches could be taken to improve and ensure they are satisfied. These include:

- providing profiles of members (to enable contact between individuals)

- suggesting related DLs and organizations, indicating attributes and differences

- providing sets of personal stories in health-support communities.

Obtaining the above types of information and keeping it current may be more of a challenge than making it available in a usable fashion. Profile information may not be necessary for all DLs, and unless there is a proven need, may require more effort that it is worth, i.e., to collect, maintain and ensure against its misuse.

At the operational level, a means of identifying specific types of messages, e.g., announcements, moderator comments, obvious flames, would aid the lurker in sorting and using the messages more effectively. A number of DLs already employ subject header prefixes for identifying message types. For this to work, members must comply with the conventions or have a moderator determine each message's type. Knowing the conventions requires an educated poster.

Goal: Satisfy information needs. Satisfying information needs was important to the participants. In some cases, information was more important than interaction. Having information in the form of archives was useful, especially if it was readily searchable. In a more passive way, the turnover of information through members' dialogue was also informative. In this way, participants were able to identify experts and if need be, seek expertise directly from these individuals.

Participants sought three types of information: factual information (e.g., job postings, and solutions to technical problems); different viewpoints arising from different levels of expertise; and access to personal experiences of others. Participants also mentioned breadth and depth of expertise as being important, as was finding "authentic" information based on an individual or group experience. Timely information was also considered quite important both in the sense of it being current, and that it meet the participants' immediate needs. Getting information from people living in the Middle East during the Gulf War was given as an example of timely information.

Professional needs, such as keeping abreast of conferences and work being done by peers and colleagues, were cited. Understanding who is doing what and where appears to be an important part of staying abreast of a professional community, particularly an academic one.

Artifacts and mechanisms for satisfying information needs must be better understood and their UIs improved. DL archives should be considered as information resources and their UIs should be designed to exploit this. Individuals within a DL act as living information sources; identifying expertise within a DL and making this identification known to members would aid information seeking. As in the previous set of design implications, message typing would be valuable for information seeking, e.g., to identify profession-related announcements. 


\subsection{Persistence as an incentive to lurk}

How does the persistence of the DL artifacts (email, digest and archives) affect public participation in DLs? What are the issues for lurkers related to persistence that keep them lurking when they might otherwise be willing to participate?

Goal: Ensure privacy and safety. Participants were generally aware that DLs have a life of their own, and that the combination of persistence and later uncontrolled access means that there is no such thing as privacy. This inhibited their posting of personal information, and in one case, a participant's employer prohibited posting. Privacy is a concern not only at the time of the posting, but also as a long term consideration due to the persistence of DL artifacts.

Members and potential members of a DL should have a clear understanding of the implications of posting, i.e., loss of privacy. Part of that understanding lies in knowing whether the DL is publicly archived, whether there are membership criteria that have to be met in order to join the DL, and whether a list of members is readily available. At this time the majority of DLs do not provide membership lists [11].

Safety is also a concern for some lurkers. Participants who had concerns about safety expressed it at two different levels. The first relates to a fear of violence, i.e., that someone or some agency can use posted information (or mere membership in a DL) to find someone or something about someone. The second relates to the fact that if you don't post you can't offend, and therefore will not become a target of flaming. While the safety issue is different from privacy, the design implications are similar.

One option for ensuring privacy and safety is the use of anonymous email hosting services such as hotmail.com. These services provide mechanisms for anonymously posting and receiving messages. There is a conundrum; participants were interested in maintaining their own privacy yet wanted to know more about other members. For example, a poster's address and signature were mentioned as a means of understanding the poster, and one participant wanted to find DL members of a similar age and gender.

Goal: Reduce noise and exposure. Most participants realized that DLs and other online forums are regularly pilfered for email addresses, which are then sold or used directly to spam. Not one participant said they look forward to receiving spams. Spammers can obtain messages directly from the messages themselves or by querying the DL server for a list of members.

As a first level of defense members' addresses should be made difficult to access. Owners of DLs can easily restrict access to the DL membership list. Similarly, DL server software can be set up to prevent the distribution of email from non-members. Some DL members take their protection one step further and provide incorrect return addresses on their email. While this may foil spamming, it makes legitimate communication difficult, e.g., to get the correct address takes more effort when side posting.

DLs allow emotional detachment as the audience and thus the lurkers are for the most part not identifiable. As one participant expressed it, when you lurk, you can have curiosity without exposure. In contrast, several participants indicated that it is much more difficult to lurk in chat rooms than DLs as chat rooms are synchronous environments where participants are normally visible and thus approachable. For some participants, the practice of lurking makes leaving a DL easier in that there is less of a commitment to a DL if you don't post. For some individuals, their notoriety makes posting problematic, e.g., few government officials post to public DLs.

Some DLs discourage lurking, at least at the outset, suggesting in their introductory message that newcomers should provide a description of themselves and post it to the DL. Other DLs specifically state that posting is not required. In either case being aware of the rules of the DL is an important part of participation. Few of the participants in this study indicated that they read the rules or guidelines.

\subsection{Persistence as overhead to lurking}

As used in this paper, overhead is defined as the set of actions and time required by the lurker to deal with DL email. For all participants, DL email was received along with other email through a single preferred email client. These email clients varied in type and configuration for each participant. As such, each participant received DL email under very different conditions. To add to the variety in overhead, their skills with the email clients ranged from naïve to expert, and the tasks they performed ranged from simple to complex.

Participants had other priorities in their lives; DL reading/following was frequently not the most important task of the day and certainly not the one in which they wished to spend most of their time, or even a good portion of their time. In the context of their lives, lurking in a DL is one of many activities filling their day. The following is a synopsis of participants' goals, their overheads and the resulting design implications.

Goal: Maximize return. In general the participants were interested in getting the most out of the time they had for lurking. Even if they lurked to entertain themselves, they still wished to do this as efficiently as possible. This typically meant spending less rather than more time with the DL(s). 
They used a number of methods to do this. If they belonged to more than one DL, they limited themselves to the number of DLs they could handle. It was clear that too many DLs meant that the value of one or more of the DLs would be reduced.

While many of the DLs described by participants had 20-30 messages/day, participants were generally happier with fewer message. Factors affecting the amount of time required to lurk on a DL include the quality and size of the messages, the motivation in belonging to the DL, the volume and type of email received from other sources, and the time available. In our examination of a number of introductory messages and DL related Web sites, none mentioned how many messages a subscriber might expect.

The asynchronous and persistent nature of DLs means that lurkers can go back through older messages at any time and either search for particular information or browse the messages.

Goal: Keep inbox manageable. Manageable meant different things to different participants, but was often related to comfort. For several participants comfort came from keeping their inbox small, i.e., able to see all retained messages at once. The process of picking through the messages was an important part of their management process. Understanding how inboxes are used is critical to developing design solutions.

The use of filters to sort messages into secondary mail boxes was not commonly used among participants. A number of reasons were stated: not trusting the effectiveness of the filters, potential burying of important email, and no knowledge of filtering tools or the process of creating effective filters. Filtering mechanisms should be examined with an aim to making them verifiable, trustworthy, and simpler to learn and use.

Goal: Identify DL email amongst other email. Differen-tiating one DL's messages from another, and those in turn from non-DL email was an effort for participants. Recognizing this as a problem, some DLs use an identifying prefix in the subject header to indicate that a message is from a particular DL, e.g., the MORE cycle DL prefixes all subject headers with "more:".

Identification of DL messages is an important mechanism for scanning and processing email in the inbox and elsewhere. The current ad hoc approach of using prefixes may be good enough, but could be improved upon. A related issue, although not raised by the participants is the use of prefixes to identify different types of messages, e.g., "Q:" for question. The use of prefixes helps identify a message's origin and intent, but may also make the subject heading more difficult to read.

Existing header information is sufficiently descriptive for use in separating messages from different DLs and
non-DL email. As mentioned in the previous goal, filtering tools remained largely unused by the participants. Whittaker and Sidner [17] found the inbox to be an important repository for messages. Their findings suggest that the low use of filters may not reside solely in the act of filtering, but on other factors, such as the fear of losing track of important information.

Goal: Follow threads. A thread is a conversation of multiple messages linked via a repeatedly used subject header. Participants were able to follow threads in newsgroups and BBSs because these systems were designed with threaded conversation in mind. Participants used threading to either follow a particular discussion or determine whether a line of discussion was worth reading. This particular facility is poorly implemented or nonexistent in most email clients. In addition, threading in email clients is different from that in newsgroups or BBSs. Even when messages can be sorted by subject header in an email client, the results are presented as a list of messages related by subject header. In both BBS and newsgroups, messages are related in a tree like manner, with the relationships between individual messages being apparent to the user. For this reason, email-based threading might better be called clumping.

For threading to be of value in email clients, threading must be effectively represented in the UI, e.g., threading based on subject header and date, and keeping the most active threads in the most visible position in order for the thread activity to remain observable to the user. GUIbased email clients can show threading based on the subject headers, but the results are frequently cumbersome and confusing. Alternative solutions need to be examined.

Additional problems occur when receiving DLs as digests. While this reduces message clutter in the inbox, it eliminates thread visibility. Current email clients are unable to show threading in digests although specialized digest readers such a Digester [16] show promise in this area.

Goal: To read or not to read. Determining what to read is an important activity for any lurker. Deciding whether a message was worth reading was idiosyncratic and for a given participant often differed between DLs. The following criteria were described:

- read all if participant is new to the DL

- read if the subject heading shows potential value

- read if the author is known

- read all messages in a thread if the middle message of a thread is interesting

- read messages if thread is long (i.e., quality of messages and thread is somehow related to the length of the thread)

- read messages with confusing subjects 
- read or not read an obvious flame.

Several participants deleted all or most messages (read or not read) as a matter of course whereas others kept messages, either by leaving them in the inbox and relying on the read flags to indicate their status, or by manually placing them in secondary folders. The delete process was most common among users of text-based email clients.

A rich set of cues were used in deciding whether to read a message. The fact that messages are persistent and asynchronous, means that a message does not have to be read at the time of receipt. It also means that the decision as to whether a particular message is read will often be based on other messages, e.g., other messages in the thread or the quantity of messages in the inbox.

\subsection{Summary}

In the three previous sections, the goal of lurkers and the corresponding effects that persistence has on those goals was outlined. In this section, the design implications are discussed based on where change would be beneficial. The following five areas are summarized below:

- email client

- server software and administration

- alternative access mechanisms

- support information

- member

There are two leading ways in which all email clients can be improved: by showing threading, and improving filtering. Threading provides lurkers with the ability to judge whether messages are valuable, and how to deal with them. It also allows the user to follow conversations more easily. At this time, threading is poorly implemented on most email clients. Filtering has the capability of separating and thus organizing multiple DLs into separate areas and thus reducing clutter in the inbox. Filtering is readily available on most GUI based email clients, but is not frequently used.

At the server level, several improvements could make life easier for the lurker. However, some improvements negatively affect other areas. For instance, digests are intended to reduce inbox clutter, especially with high volume lists. However, thread following is compromised when messages are delivered in digest form. There is some evidence [11] that DLs that are set to send out digests to new subscribers also have higher levels of lurking. Whether this is a result of digests being less well read, more difficult to respond to, or harder to follow threads is not known.

Many DLs add a prefix to the subject header as a means of identifying messages. These prefixes may make DL identification easier, but likely obscure the actual subject header. Whether knowing that a message comes from a particular DL is more important than the subject is unclear. It will likely depend on many factors, including the volume of messages in the inbox, the rate of receipt, and purpose of belonging to the DL.

Most DL administrators prevent access to membership lists. More often than not they also prevent messages being broadcast by non-members. There is however, very little they can do to prevent the pilfering of addresses from archives. Some members have taken up the antispam challenge by supplying incorrect return addresses.

At the level of supporting the lurker with information related to the DL, providing an accessible, current, and usable set of information is important. Creating links to it in all outgoing messages would provide access. Within the Web site or wherever it may reside, access to an archive is an important information resource for many lurkers, particularly if they are trying to understand the nature of the DL, or looking for specific information. A usable interface should allow lurkers to browse, follow threads and search for information.

An archive can also provide the functionality for posting messages. This can either reduce or eliminate the reliance on the email client. In doing so, many of the problems described so far could be reduced, e.g., threading is usually apparent in Web-based interfaces, anonymity is frequently built into the system ensuring safety and privacy, and an archive and supporting information can be integrated into the environment.

On the down side, the user may have a different identity and potentially a different password for each DL they belong to. UIs while similar in intent between DLs will be different and will require familiarization with each. In contrast, email-based DLs utilize a single familiar UI for a given user. In addition, the email client can receive email automatically, whereas, Web-based DLs rely on the user to seek them out.

For the security conscious, the use of Web-based DLs may offer a preferred solution. However, it is unclear whether security is more important than the convenient and consistent albeit somewhat underused and noisy UI of the email client.

Email clients may be facing functionality bloat already, adding additional functionality for lurking may not be the best approach. Improved lurking may come through improving the skills of the lurker. While the current email clients may not have been specifically designed with lurking and DLs in mind, many of their facilities go unused by the lurker, e.g., use of secondary mail boxes and filtering. This is in part due to users not being familiar with the functionality of the email client, but also stems from the way in which they view and use the inbox as a central repository. As is the case with other software, DL members use the tool to the extent that fulfills their immediate needs. Its likely that the level of participation (posting or not) in one or more DLs is a 
function of their skills in using the email client. Other factors will also be at work, such as volume of email, personal strategies, motivation, time available, etc. By improving our understanding of strategies and the context in which the strategies develop, we should be able to come up with a better model of the lurker, and improvements in their tools.

\section{Conclusion}

As a means for asynchronous group communication, DLs have gained wide acceptance. This is in part due to their use of the most ubiquitous of Internet tools, the email client. We have illustrated a number of issues and design implications related to persistence.

As aid to their lurking, participants described how they followed online conversations to understand the practices and language of a DL. They talked about observing the coming out of other lurkers, and measuring the group by its treatment of new members. Several participants developed a sense of community in the process of following conversational threads. As an incentive to lurking, security and privacy are very important issues. Participants viewed persistent conversation, especially when it can be retrieved through search mechanisms at a later date, as an impediment to public participation. As overhead to lurking, participants described the process of using and maintaining their DL email. Mechanisms for reading and managing email were idiosyncratic and goal driven. Some participants were concerned about maintaining access to found information for future use. Others, more concerned about being overloaded with too much email, focused on eliminating messages while getting the most out of what they could make use of in the moment.

It is not surprising that some of the lurkers' goals lead to contradictory design implications. On one hand, privacy is a very important issue, and on the other, participants desired more information about the DL and its members. Email clients are relatively simple, well understood tools which in part accounts for the popularity of DLs. Improving in their facility as DL front ends may increase their complexity and thus compromise their broad appeal. Full featured Web based UIs to DLs hold promise for eliminating many of the problems associated with the email-based UI. Whether lurkers are willing to switch to an alternative UI is unknown.

The method of using a small group of participants, interviewing them with regard to the membership and practices within online groups was an effective technique for exposing a wide variety of issues related to lurking. We now have a good base for carrying out in-depth interviews and surveys in order to understand the relevance of the findings and the usefulness of the design implications.
The design implications coming from this work should be of interest to developers of email clients. This work will be followed by evaluations of several email clients and at least one digest reader, with an eye to examining how the tools have shaped usage, and how current usage can improve the design of the tools. While this work has focused on lurking, the issues raised are applicable to all DL members regardless of whether they post or not. There will be additional specific issues and design implications for public posting in DLs.

\section{Acknowledgments}

This work would not have been possible without our interviewees; we thank them for their time and insight. Carolyn Davidson and Heather McDonald provided thought provoking and valuable reviews of early drafts.

\section{References}

[1] Excite.com. What We Do. Available at http://corp. excite.com/Company/what.html.

[2] Holtzblat, K., and H. Beyer. Contextual Design: using customer work models to drive system design. Proceedings of ACM CHI'95 Conference on Human Factors in Computing Systems, 1995), 391-392.

[3] Jargon-Dictionary. Lurker Definition. Available at http://www.netmeg.net/jargon.

[4] Katz, J. Luring the Lurkers. Available at http:// slashdot.org/features/98/12/28/1745253.shtml.

[5] Kollock, P., and M. Smith. Introduction: Communities in Cyberspace. In: Communities in Cyberspace, edited by P. Kollock and M. Smith. London: Routledge, 1999.

[6] Kollock, P., and M. Smith. Managing the virtual commons: cooperation and conflict in computer communities. In: Computer-Mediated Communication: Linguistic, Social, and Cross-Cultural Perspectives, edited by S. Herring. Amsterdam: John Benjamins, 1996, 109-128.

[7] L-Soft International. Record sites. Available at http:// www.lsoft.com/listserv.stm.

[8] Mason, B. Issues in virtual ethnography. Ethnographic Studies in Real and Virtual Environments: Inhabited Information Spaces and Connected Communities (Edinburgh, 1999), 61-69.

[9] Merriam-Webster. Lurk. Available at http://www.mw.com/cgi-bin/dictionary?book=Dictionary \&va $=$ lurk. 
[10]Nielsen, J. Usability Engineering: Academic Press, Boston, 1993.

[11] Nonnecke, B. Lurking in email-based discussion lists. In: SCISM. London: South Bank University, 2000.

[12] Nonnecke, B., and J. Preece. Shedding light on lurkers in online communities. Ethnographic Studies in Real and Virtual Environments: Inhabited Information Spaces and Connected Communities (Edinburgh, 1999), 123-128.

[13]Parks, R. M., and K. Floyd. Making friends in cyberspace. Journal of Computer-Mediated Communication 1, 4, (1996).
[14]Preece, J. Empathic Communities: Balancing emotional and factual communication. Interacting with Computers: The interdisciplinary Journal of HumanComputer Interaction 5, 2, (1998), 32-43.

[15] Smith, C. B. Casting the net: Surveying an Internet population. Journal of Computer-Mediated Communication 3, 3, (1997).

[16] TECHWR-L. About the Digest Reader. Available at http://www.raycomm.com/techwhirl/digestreader.html.

[17] Whittaker, S., and C. Sidner. Email overload: Exploring personal information management of email. CHI'96 (Vancouver, BC, 1996), ACM Press, 276-283. 